\title{
The Correlations Between the Expression of SSTR2, SSTR5 Proteins and Clinicopathological Parameters as well as Prognosis of Gastric Neuroendocrine Neoplasms
}

\section{Yanwei Ye ( $\nabla$ yeyanwei66@163.com)}

Departments of Gastrointestinal Surgery and Institute of Clinical Medicine, The First Affiliated Hospital of Zhengzhou University

\section{Chuangfeng Xiao}

Departments of Gastrointestinal Surgery and Institute of Clinical Medicine, The First Affiliated Hospital of Zhengzhou University; Zhengzhou, Henan 450052, P.R. China

\section{Yingze Li}

Departments of Gastrointestinal Surgery and Institute of Clinical Medicine, The First Affiliated Hospital of Zhengzhou University; Zhengzhou, Henan 450052, P.R. China

\section{YiMing Shan}

Departments of Gastrointestinal Surgery and Institute of Clinical Medicine, The First Affiliated Hospital of Zhengzhou University; Zhengzhou, Henan 450052, P.R. China

Jie Li

Department of General Surgery. Tongchuan People's Hospital. Shanxi 727000, P.R. China

\section{Dongbao Jiang}

Department of thyroid, and breast surgery, Xinxiang Central Hospital, Xinxiang, Henan 453000, P.R.

China

Jingjing Li

Department of Infectious Disease, The First Affiliated Hospital of Zhengzhou University; Zhengzhou, Henan 450052, P.R. China

\section{Chao Han}

Department of Pharmacy, The First Affiliated Hospital of Zhengzhou University; Zhengzhou, Henan 450052, P.R. China

\section{Wencai Li}

Department of Pathology, The First Affiliated Hospital of Zhengzhou University; Zhengzhou, Henan 450052, P.R. China

\section{Research}

Keywords: SSTR2, SSTR5, gastric neuroendocrine neoplasms, G-NENs, Prognosis 
Posted Date: September 8th, 2020

DOl: https://doi.org/10.21203/rs.3.rs-66797/v1

License: (c) (i) This work is licensed under a Creative Commons Attribution 4.0 International License. Read Full License 


\section{Abstract}

Background囚 Somatostatin receptor 2, 5 (SSTR2, SSTR5) were seldom investigated in gastric neuroendocrine neoplasms (G-NENs). The purpose of the study was to elucidate the expression of SSTR2, SSTR5 in G-NENs and related clinical significance.

Methods 66 paraffin-embedded specimens were obtained from The first affiliated hospital of Zhengzhou university. The expression of SSTR2 and SSTR5 was detected by immunohistochemistry. The expression of SSTR2, SSTR5 and the clinicopathological characteristics, related immunohistochemical molecules and prognosis of gastric neuroendocrine neoplasm were analyzed statistically.

Results $\llbracket$ The expression rate of SSTR2 protein in G-NENs tissues and normal stomach tissues was $48.5 \%$ and $25.0 \%$, respectively $(\mathrm{P}=0.046)$; the expression rate of SSTR5 protein in G-NENs tissues and normal stomach tissues was $65.2 \%$ and $25.0 \%$, respectively $(P=0.018)$. The expression of SSTR2 was positively correlated with the expression of Ki-67, SSTR5 and tumor grade (P-value was $0.032,0.002$, and 0.005 , respectively); the expression of SSTR5 was positively correlated with the expression of SSTR2, Ki-67, CD56 and tumor grade (P-value was $0.032,0.011,0.008,0.028$, respectively). In the SSTR2-positive group, SSTR5, CD-56, Ki-67 were closely related to the prognosis of patients with G-NENs. In the SSTR5-positive group, tumor grade, SSTR2, CD-56, Ki-67 were closely related to the prognosis of patients with G-NENs. Multi-factor analysis showed that SSTR2 and SSTR5 were independent prognostic factors for patients with G-NENs.

Conclusion. High expression of SSTR2 and SSTR5 protein was related to the tumorigenesis of G-NENs. SSTR2 and SSTR5 were associated with the prognosis and might improve the prognosis of G-NENs.

\section{Background}

The Somatostatin receptor (SSTR) family with its closely five related human members (SSTR1-SSTR5) is a $\mathrm{G}$ protein-coupled receptor encoded by five different genes on a single chromosome and is a glycoprotein with seven transmembrane segments[1,2].The distribution of SSTRs was universal and abundant in all tissues of the human body. Somatostatin mainly exists in the central nervous system, pancreas and gastrointestinal tract, which has a wide range of inhibitory functions, including the inhibition of hypothalamic hormone, gastrin and gastric acid secretion, insulin release and so on [3-5]. It has also been shown to inhibit cell proliferation and have anti-inflammatory effects. These properties make somatostatin a potential candidate for the treatment of many diseases. Currently, it is commonly used to treat acromegaly, Cushing's disease and neuroendocrine neoplasm. The effect of somatostatin on different tissues depends on the type of somatostatin receptor (SSTR) expressed on the surface. Wang Y's study showed that SSTR was overexpressed in gastrointestinal pancreatic neuroendocrine neoplasm, especially the expression of SSTR2 and SSTR5; Positive expressions of SSTR2 and SSTR5 can predict the survival rate of patients with GEP-NENs; and the expression of various SSTRS on tumor cells constitutes the basis of somatostatin treatment for patients with neuroendocrine neoplasm [6]. 
At present, the expression and mechanism of SSTR subtypes in gastric neuroendocrine neoplasm have not been fully confirmed and the therapeutic effect varies greatly. It is reasonable to speculate that the poor therapeutic effect of somatostatin analogues is related to the loss of endogenous somatostatin receptor expression or the expression amount and subtype combination. So it is particularly necessary to deeply explore the relationship between SSTRs and gastric neuroendocrine neoplasm.

Therefore, the purpose of this study was to detect the expression of SSTR2 and SSTR5 in 66 G-NENs tissues with complete followup information as well as 12 normal stomach tissues by immunohistochemical staining. Combining patients' clinicopathological characteristics and follow-up data, correlation analysis and prognostic analysis were carried out to verify whether the expression of SSTR2 and SSTR5 protein had clinical significance. Furthermore, some common molecular markers of GNENs in our cancer center, including CK-8, CD-56, CgA, Syn, and Ki-67, were detected by immunohistochemical staining and introduced to analyze their association with the expression of SSTR2 and SSTR5 protein. We expected that SSTR2 and SSTR5 might provide new ideas and methods for the diagnosis and treatment of this tumor.

\section{Methods}

\section{Patients and Tissue samples}

Paraffin-embedded tissue specimens of 66 consecutive primary G-NENs and 12 normal stomach tissues (more than $5 \mathrm{~cm}$ away from tumor border) were collected by the first affiliated hospital of Zhengzhou university (Zhengzhou, China). All patients were diagnosed and treated at the Department of Gastrointestinal surgery in 2015-2018. 66 G-NENs patients had not undergone any chemotherapy, radiotherapy, and other treatments before radical gastrectomy were performed. All cases were pathologically documented and personal files were recorded to obtain clinical data,under the approval of the hospital's ethical committee. SPSS 22.0 was used to collect and sort patients' clinicopathological and follow-up data, including gender, age, location of tumors, tumor size, invasion depth, lymph node status, nerve and vessel invasion, and follow-up data. The classification of gastric neuroendocrine neoplasms was based on the WHO classification and classification standards of digestive system tumors in 2010 [7]. Follow-up data were obtained by phone, letter, and the outpatient clinical database. Survival time was calculated from the date when surgery was done to the date of death or the date of the last followup. The follow-up period was closed in march 31,2020. A followup of all patients was carried out according to our standard protocol (every 3 months for at least 2 years, every 6 months for the next 3 years, and after 5 years every 12 months for life). Median follow-up in patients still alive at analysis was 36.5 months (range, 12-54 months).

\section{Immunohistochemical Staining}


SSTR2, SSTR5, CK-8, CD-56, CgA, Syn, and Ki-67 expression in postoperative paraffin-embedded tumor specimens of all selected patients were detected by immunohistochemical method. The concentration of antibodies and positive site were as follows: anti-SSTR2, dilution 1:100, positive site is cytoplasm or membrane; anti-SSTR5, dilution 1:50, positive site is cytoplasm or membrane; anti-CK-8, dilution1:100, positive site is cytoplasm; anti-CD-56, dilution 1:50, positive site is membrane; Anti-CgA, dilution 1:50, positive site is cytoplasm; anti-Syn, dilution 1:50, positive site is cytoplasm; anti-Ki-67, dilution 1:100, and positive site is nucleus. The detailed staining procedures were strictly followed the supplier's recommendation. Negative controls were obtained by incubation of parallel slides omitting the primary antibodies. Besides, sections known to be stained positively in each run were served as positive controls.

\section{Immunohistochemical Staining Scoring}

Slides were all semi-quantitatively evaluated by two independent pathologists (L. W.C.and H.H.Y), who were blinded to patients' clinical data when scoring immunohistochemical results of the archival tissue samples. The initial score was divided into four levels by the range of cell staining: -, no cell staining or range $<10 \% ;+, 11-25 \%$ staining;,$++ 26-50 \%$ staining;,$+++ 50 \%>$ staining. The expressions of SSTR2 and SSTR5 are mainly cytoplasmic staining. By semi-quantitative analysis, the immunostaining of SSTR2 and SSTR5 can be divided into four grades, negative (-), low (+), intermediate (++), and high expression (+++). When the proportion of Ki-67 positive cells was calculated, 500-2000 cells were counted in the most strongly labeled and stained area under at least 50 microscope high-power field (1 high-power field $=2 \mathrm{~mm}$ ), and the proportion of Ki-67 positive cells in tumor cells (i.e., Ki-67 index) was calculated. In the final statistical analysis, the negative staining of CK-8, CD-56, CgA, and Syn was defined as the tumor cytoplasm staining $\leq 10 \%$. Positive staining of CK-8, CD-56, CgA and Syn was defined as $>10 \%$ staining of tumor cytoplasm or cell membrane. The above diagnostic criteria refer to the product specification and related literature of zhongshan jinqiao company.

\section{Statistical Analysis}

The association between SSTR2, SSTR5 expression and clinicopathological factors as well as other immunohistochemical markers was evaluated by chi-square test. The 3-year survival rate was calculated by the Life Tables method, survival curves were obtained by Kaplan-Meier method and differences between survival curves were examined with the log-rank test. All the statistically significant variables observed in univariate analysis were investigated by means of multivariate analysis using the Cox proportional hazards model. All statistical tests were two-sided, and significance was set at the 0.05 level. Statistical analysis and graphics were performed by the SPSS 22.0 statistical software package (SPSS, Chicago, IL).

\section{Results}




\section{Clinicopathological Characteristic of Gastric neuroendocrine neoplasms Patients}

66 G-NENs patients have been recruited in this study, 50 males and 16 females; 36 patients with $\leq 60$ years while 30 patients with more than 60 years at the data of diagnosis; 47 patients located in the cardia and body of gastric and 19 patients located in the gastric antrum; the tumor size of 23 patients were $<4 \mathrm{~cm}$ while 43 patients had tumor size $\geq 4 \mathrm{~cm} ; 10$ patients with tumors invaded the mucosa and submucosa, 12 patients with tumors invaded the muscularis and 39 cases patients with tumors invaded the serosa; 22 cases without lymph node metastasis while 40 cases with it; patients with or without vessel and nerve invasion were 28 and 15 cases, respectively; furthermore, 10 patients with $\mathrm{G} 1,16$ patients with G2, 8 patients with G3 NEN and 32 patients with G3.

\section{Expression of Markers in Gastric neuroendocrine neoplasms}

66 paraffifin-embedded G-NENs tissues and 12 normal stomach specimens were all selected from the Department of pathology, the first affiliated hospital of Zhengzhou university in 2019.

SSTR2 expression in G-NENs tissues were as follows: - , 34 cases $(51.5 \%) ;+, 10$ cases $(15.2 \%) ;++, 15$ cases $(22.7 \%) ;+++, 7$ cases $(10.6 \%)$, respectively. SSTR2 expression in normal stomach tissues from - to +++ were 9 cases $(75.0 \%), 0$ cases $(0 \%), 3$ case $(25.0 \%)$, and 0 case $(0 \%)$, respectively. Therefore, the positive expression of SSTR2 in G-NENs tissue and normal stomach tissue were $48.5 \%$ and $25.0 \%$, respectively. Pearson $\chi^{2}$ test showed that SSTR2 expression in two groups had significant different ( $\chi^{2}$ value $=4.00, P=0.046$ ). In other word, positive expression of SSTR2 protein existed in G-NENs tissue. Figure $1 \mathrm{a}$ and $1 \mathrm{~b}$ illustrated the positive expression and negative of SSTR2 in the cytoplasm of GNENs cells, respectively; Figure 1c and 1d showed that the positive expression and negative of SSTR2 in the cytoplasm of normal stomach tissue cells, respectively.

SSTR5 expression in G-NENs tissues were as follows: -, 23 cases $(34.8 \%) ;+, 13$ cases $(19.7 \%) ;++, 22$ cases (33.3\%); +++, 8 cases (12.2\%), respectively. SSTR5 expression in normal stomach tissues from - to +++ were 9 cases $(75.0 \%), 1$ cases $(8.3 \%), 2$ case $(16.7 \%)$, and 0 case $(0 \%)$, respectively. Therefore, the positive expression of SSTR5 in G-NENs tissue and normal stomach tissue were $65.2 \%$ and $25.0 \%$, respectively. Pearson $\chi^{2}$ test showed that SSTR5 expression in two groups had significant different ( $\chi^{2}$ value $=5.60, P=0.018$ ). In other word, the positive expression of SSTR5 protein existed in G-NENs tissue. Figure $1 \mathrm{e}$ and $1 \mathrm{f}$ illustrated the positive expression and negative of SSTR5 in the cytoplasm of GNENs cells, respectively; Figure $1 \mathrm{~g}$ and $1 \mathrm{~h}$ showed the positive expression and negative of SSTR5 in the cytoplasm of normal stomach tissue cells, respectively.

Furthermore, just as Table 1 displayed, the positive rate of CK-8, CD-56, CgA and Syn expression in GNENs tissues were $82.0 \%$ (50/61), 75.8\%(50/66), 65.9\% (29/44) and 97.0\% (64/66), respectively. The expression of Ki-67 was more than $20 \%$ in $60.6 \%$ (40/66) G-NENs tissues. 
Table 1

Correlation Between SSTR2 Expression and Clinicopathologic Characteristic of Gastric neuroendocrine neoplasms Patients

\begin{tabular}{|c|c|c|c|c|}
\hline Variable & $\mathbf{n}$ & Positive group ${ }^{a}$ & Negative group ${ }^{b}$ & $P$-value \\
\hline ALL cases & 66 & 32 & 34 & \\
\hline Age(years) & & & & 0.208 \\
\hline$\leq 60$ & 36 & 20 & 16 & \\
\hline$>60$ & 30 & 12 & 18 & \\
\hline \multicolumn{5}{|l|}{ Gender } \\
\hline Male & 50 & 23 & 27 & 0.475 \\
\hline Female & 16 & 9 & 7 & \\
\hline Tumor site & & & & 0.668 \\
\hline Cardia and body of gastric & 47 & 22 & 25 & \\
\hline Gastric antrum & 19 & 10 & 9 & \\
\hline Tumor size & & & & 0.141 \\
\hline$\otimes 4 \mathrm{~cm}$ & 23 & 14 & 9 & - \\
\hline$\geq 4 \mathrm{~cm}$ & 43 & 18 & 25 & \\
\hline Invasion depth & & & & 0.213 \\
\hline Mucosa and submucosa & 10 & 5 & 5 & \\
\hline Muscularis & 12 & 3 & 9 & \\
\hline Serosa & 39 & 21 & 18 & \\
\hline Lymph node metastasis & & & & 0.160 \\
\hline Yes & 40 & 22 & 18 & \\
\hline No & 22 & 8 & 14 & \\
\hline Vessel and nerve invasion & & & & 0.284 \\
\hline Yes & 28 & 12 & 16 & \\
\hline No & 15 & 9 & 6 & \\
\hline Tumor grade & & & & 0.005 \\
\hline $\mathrm{G} 1$ & 10 & 6 & 4 & \\
\hline
\end{tabular}




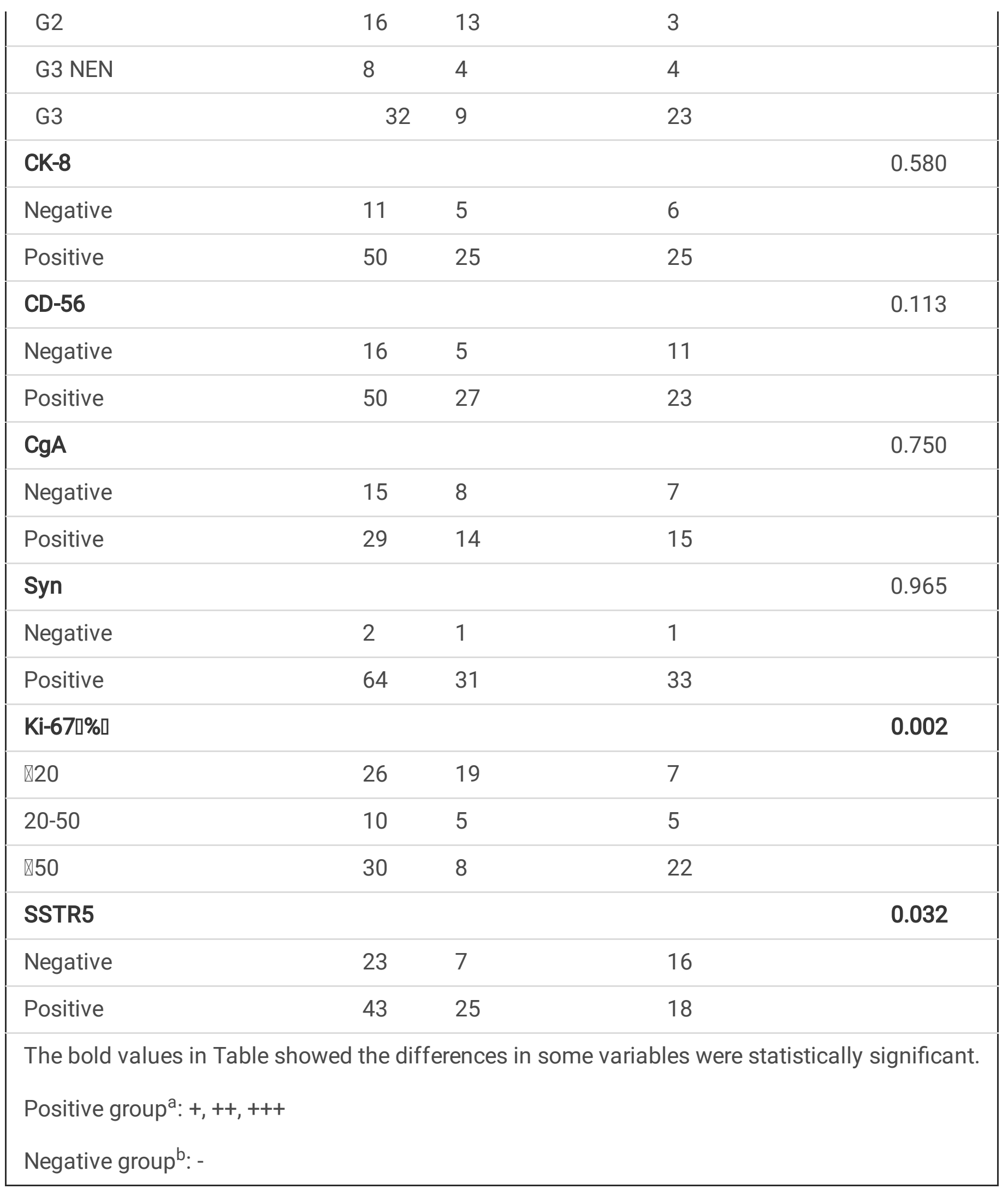

\section{Correlations Between SSTR2, SSTR5 Expression and Clinicopathological Parameters}


To explore the association of SSTR2, SSTR5 expression and clinicopathological characteristics, 66 immunohistochemical slides of G-NENs tissues were divided into two groups for analysis, a negative group (including - ) and a positive group of SSTR2and SSTR5 (including,,++++++ ).

Just as Table 1 revealed, there were no significant differences between the positive group and the negative group of SSTR2 involving in some clinicopathological parameters and molecular markers, including age, gender, tumor location, tumor size, invasion depth, lymph node metastasis, vessel and nerve invasion as well as the expression of CK-8, CD-56, CgA and Syn $(P>0.05)$. There were statistically significant differences in tumor grade, Ki-67 and SSTR5 in the SSTR2 expression group $(P=0.005,0.002$ and 0.032 , respectively).

Just as Table 2 revealed, there were no significant differences between the positive group and the negative group of SSTR5 involving in some clinicopathological parameters and molecular markers, including age, gender, tumor location, tumor size, invasion depth, lymph node metastasis, vessel and nerve invasion as well as expression of CK-8, CgA and Syn ( $P>0.05)$. There were statistically significant differences in tumor grade, CD-56, Ki-67 and SSTR2 in the SSTR5 expression group $(P=0.028,0.008$, 0.011 and 0.032 , respectively). 
Table 2

Correlation Between SSTR5 Expression and Clinicopathologic Characteristic of Gastric neuroendocrine neoplasms Patients

\begin{tabular}{|c|c|c|c|c|}
\hline Variable & $\mathrm{n}$ & Positive group ${ }^{a}$ & Negative group ${ }^{b}$ & $P$-value \\
\hline ALL cases & 66 & 43 & 23 & \\
\hline Age(years) & & & & 0.187 \\
\hline$\leq 60$ & 36 & 26 & 10 & \\
\hline$>60$ & 30 & 17 & 13 & \\
\hline \multicolumn{5}{|l|}{ Gender } \\
\hline Male & 50 & 33 & 17 & 0.798 \\
\hline Female & 16 & 10 & 6 & \\
\hline Tumor site & & & & 0.829 \\
\hline Cardia and body of gastric & 47 & 31 & 16 & \\
\hline Gastric antrum & 19 & 12 & 7 & \\
\hline Tumor size & & & & 0.593 \\
\hline$₫ 4 \mathrm{~cm}$ & 23 & 14 & 9 & - \\
\hline$\geq 4 \mathrm{~cm}$ & 43 & 29 & 14 & \\
\hline Invasion depth & & & & 0.837 \\
\hline Mucosa and submucosa & 10 & 6 & 4 & \\
\hline Muscularis & 12 & 7 & 5 & \\
\hline Serosa & 39 & 26 & 13 & \\
\hline Lymph node metastasis & & & & 0.508 \\
\hline Yes & 40 & 27 & 13 & \\
\hline No & 22 & 13 & 9 & \\
\hline Vessel and nerve invasion & & & & 0.606 \\
\hline Yes & 28 & 19 & 9 & \\
\hline No & 15 & 9 & 6 & \\
\hline Tumor grade & & & & 0.028 \\
\hline G1 & 10 & 7 & 3 & \\
\hline
\end{tabular}




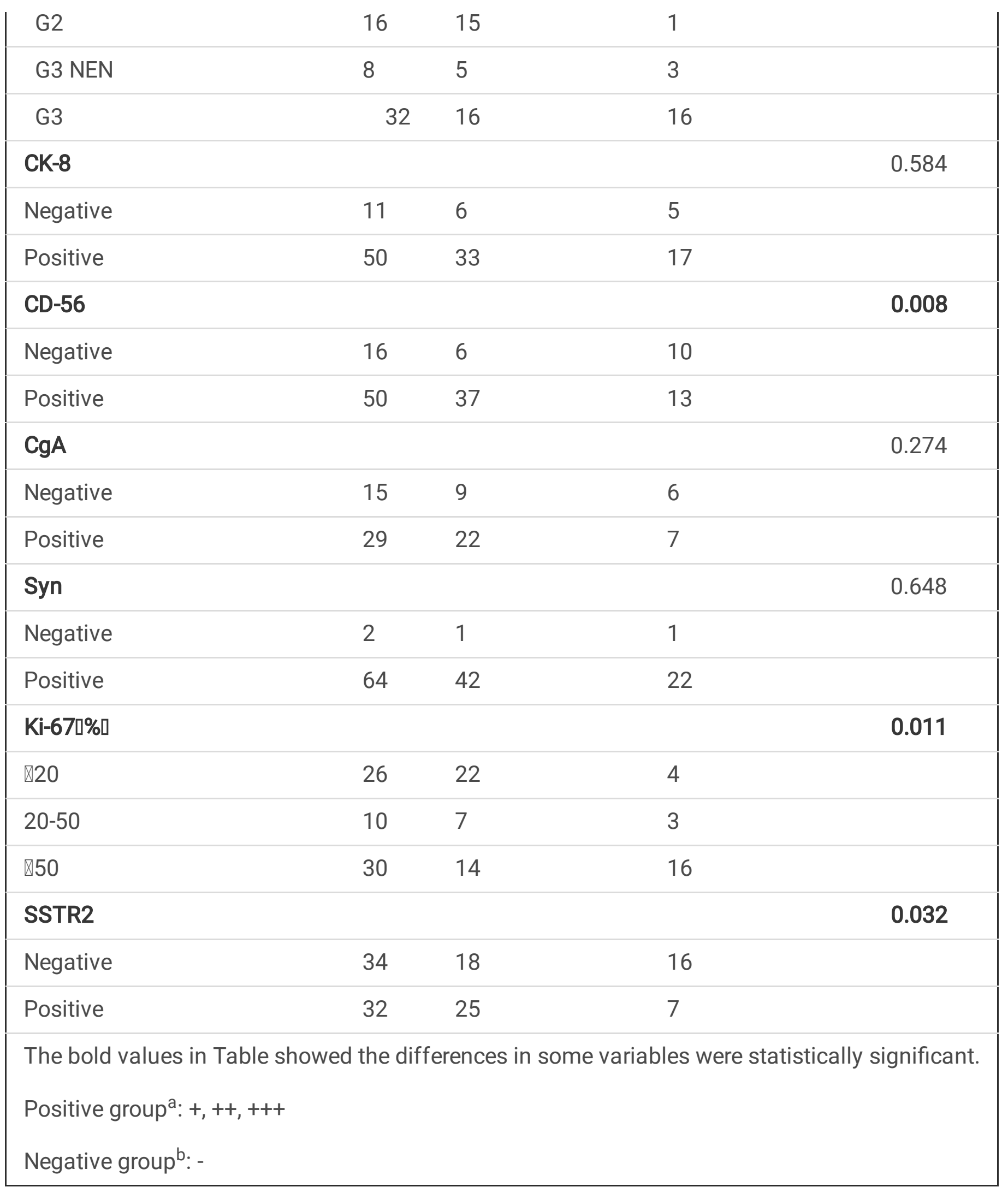

\section{Prognostic Analysis of Patients with Gastric neuroendocrine neoplasms in the Positive and Negative Groups of SSTR2 and SSTR5}


To clarify whether the expression of SSTR2 and SSTR5 protein influenced the prognosis of G-NENs patients, combining follow-up data, survival discrepancy in the high and low expression groups of SSTR5 were revealed by Kaplan-Meier method and log rank test (Fig. 2 and Table 3, 4). Furthermore, life table method was used to calculate the 3-year survival rate. 
Table 3

Survival Analysis Between Positive and Negative Group of SSTR2 in Gastric neuroendocrine neoplasms by Kaplan-Meier Method (Log-Rank Test)

\begin{tabular}{|c|c|c|c|c|c|c|}
\hline \multirow[t]{2}{*}{ Variable } & \multicolumn{3}{|c|}{ Positive group ${ }^{a}$} & \multicolumn{3}{|c|}{ Negativegroup ${ }^{b}$} \\
\hline & $\mathrm{n}$ & $\begin{array}{l}\text { 3-year survival } \\
\text { rate }\end{array}$ & $\begin{array}{l}P- \\
\text { value }\end{array}$ & $\mathrm{n}$ & $\begin{array}{l}\text { 3-year survival } \\
\text { rate }\end{array}$ & $\begin{array}{l}P \text { - } \\
\text { value }\end{array}$ \\
\hline Age(years) & & & 0.262 & & & 0.078 \\
\hline$\leq 60$ & 20 & 85 & & 16 & 75 & \\
\hline$>60$ & 12 & 91 & & 18 & 50 & \\
\hline Gender & & & 0.121 & & & 0.496 \\
\hline Male & 23 & 91 & & 27 & 56 & \\
\hline Female & 9 & 78 & & 7 & 71 & \\
\hline Tumor site & & & 0.770 & & & 0.862 \\
\hline $\begin{array}{l}\text { Cardia and body of } \\
\text { gastric }\end{array}$ & 22 & 82 & & 25 & 60 & \\
\hline Gastric antrum & 10 & 90 & & 9 & 55 & \\
\hline Invasion depth & & & 0.130 & & & 0.052 \\
\hline Mucosa and submucosa & 5 & 64 & & 5 & 100 & \\
\hline Muscularis & 3 & 67 & & 9 & 78 & \\
\hline Serosa & 21 & 36 & & 18 & 33 & \\
\hline Lymph node metastasis & & & 0.976 & & & 0.654 \\
\hline Yes & 22 & 86 & & 18 & 56 & \\
\hline No & 8 & 88 & & 14 & 64 & \\
\hline $\begin{array}{l}\text { Vessel and nerve } \\
\text { invasion }\end{array}$ & & & 0.319 & & & 0.104 \\
\hline Yes & 9 & 89 & & 16 & 75 & \\
\hline No & 12 & 100 & & 6 & 67 & \\
\hline Tumor grade & & & 0.079 & & & 0.531 \\
\hline G1 & 6 & 83 & & 4 & 75 & \\
\hline G2 & 13 & 92 & & 3 & 67 & \\
\hline G3 NEN & 4 & 75 & & 4 & 50 & \\
\hline
\end{tabular}




\begin{tabular}{|c|c|c|c|c|c|c|}
\hline G3 & 9 & 89 & & 23 & 57 & \\
\hline SSTR5 & & & 0.000 & & & 0.003 \\
\hline Negative & 7 & 57 & & 16 & 63 & \\
\hline Positive & 25 & 96 & & 18 & 67 & \\
\hline CK-8 & & & 0.639 & & & 0.912 \\
\hline Negative & 5 & 80 & & 6 & 67 & \\
\hline Positive & 25 & 88 & & 25 & 56 & \\
\hline CD-56 & & & 0.000 & & & 0.029 \\
\hline Negative & 5 & 60 & & 11 & 36 & \\
\hline Positive & 27 & 93 & & 19 & 70 & \\
\hline CgA & & & 0.606 & & & 0.943 \\
\hline Negative & 8 & 88 & & 7 & 71 & \\
\hline Positive & 14 & 86 & & 15 & 60 & \\
\hline Syn & & & 0.744 & & & 0.352 \\
\hline Negative & 1 & 100 & & 1 & 76 & \\
\hline Positive & 21 & 62 & & 33 & 64 & \\
\hline Ki-67ロ\%口 & & & 0.01 & & & 0.287 \\
\hline$₫ 20$ & 19 & 75 & & 7 & 71 & \\
\hline $20-50$ & 5 & 57 & & 5 & 60 & \\
\hline$\varangle 50$ & 8 & 54 & & 22 & 55 & \\
\hline G3 & 9 & 89 & & 23 & 57 & 0.037 \\
\hline SSTR2 Negative/Positive & 32 & 82 & & 34 & 35 & 0.002 \\
\hline \multicolumn{2}{|l|}{ Positive group ${ }^{\mathrm{a}}:+,++,+++$} & & & & & \\
\hline Negative group ${ }^{\text {b: - }}$ & & & & & & \\
\hline
\end{tabular}


Table 4

Survival Analysis Between Positive and Negative Group of SSTR5 in Gastric neuroendocrine neoplasms by Kaplan-Meier Method (Log-Rank Test)

\begin{tabular}{|c|c|c|c|c|c|c|}
\hline \multirow[t]{2}{*}{ Variable } & \multicolumn{3}{|c|}{ Positive group ${ }^{a}$} & \multicolumn{3}{|c|}{ Negative group ${ }^{b}$} \\
\hline & $\mathbf{n}$ & 3-year survival rate & P-value & $\mathrm{n}$ & $\begin{array}{l}\text { 3-year survival } \\
\text { rate }\end{array}$ & $\begin{array}{l}P \text { - } \\
\text { value }\end{array}$ \\
\hline Age(years) & & & 0.885 & & & 0.187 \\
\hline$\leq 60$ & 26 & 85 & & 10 & 70 & \\
\hline$>60$ & 17 & 71 & & 13 & 54 & \\
\hline Gender & & & 0.208 & & & 0.084 \\
\hline Male & 33 & 82 & & 17 & 53 & \\
\hline Female & 10 & 78 & & 6 & 83 & \\
\hline Tumor site & & & 0.663 & & & 0.959 \\
\hline Cardia and body of gastric & 31 & 77 & & 16 & 56 & \\
\hline Gastric antrum & 12 & 83 & & 7 & 71 & \\
\hline Invasion depth & & & 0.461 & & & 0.052 \\
\hline Mucosa and submucosa & 6 & 100 & & 4 & 75 & \\
\hline Muscularis & 7 & 71 & & 5 & 60 & \\
\hline Serosa & 26 & 73 & & 13 & 54 & \\
\hline Lymph node metastasis & & & 0.797 & & & 0.462 \\
\hline Yes & 27 & 81 & & 13 & 54 & \\
\hline No & 13 & 85 & & 9 & 67 & \\
\hline Vessel and nerve invasion & & & 0.802 & & & 0.174 \\
\hline Yes & 19 & 89 & & 9 & 78 & \\
\hline No & 9 & 89 & & 6 & 33 & \\
\hline Tumor grade & & & 0.012 & & & 0.536 \\
\hline G1 & 6 & 86 & & 3 & 67 & \\
\hline G2 & 15 & 93 & & 1 & 0 & \\
\hline G3 NEN & 5 & 60 & & 3 & 67 & \\
\hline
\end{tabular}


SSTR2

Negative

Positive

CK-8

Negative

Positive

CD-56

Negative

Positive

CgA

Negative

Positive

Syn

Negative

Positive

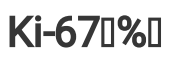

$\otimes 20$

20-50

$\varangle 50$

G3

SSTR5
$6 \quad 83$

$33 \quad 84$

$7 \quad 57$

$25 \quad 96$

0.856

0.002

0.110

$16 \quad 63$

$18 \quad 67$

0.889

$5 \quad 60$

$17 \quad 59$

0.000

0.923

$\begin{array}{ll}6 & 17\end{array}$

$10 \quad 60$

$37 \quad 89$

$13 \quad 62$

0.082

0.229

$\begin{array}{llll}9 & 100 & 6 & 50 \\ 22 & 77 & 7 & 86\end{array}$

0.622

0.244

$1 \quad 100$

10

$42 \quad 62$

$22 \quad 59$

0.001

0.767

$22 \quad 91$

$4 \quad 50$

$5 \quad 71$

$\begin{array}{ll}3 & 67\end{array}$

$14 \quad 64$

$10 \quad 63$

$16 \quad 56$

$16 \quad 63$

0.001

$32 \quad 83$

$34 \quad 76$

0.000

Negative/Positive

The bold values in Table showed the differences in some variables were statistically significant.

Positive group ${ }^{\mathrm{a}}:+,++,+++$

Negative group ${ }^{b}$ : -

In the SSTR2-positive group, the 3-year survival rate of SSTR5-positive group was 96\%, which was significantly higher than that of SSTR5-negative group (57\%) and the difference was statistically significant (Fig. 2a, $P=0.000$ ). The 3-year survival rates for the CD-56 positive group and negative group 
were $93 \%$ and $60 \%$, respectively (Fig. $2 \mathrm{~b}, \mathrm{P}=0.000$ ). Moreover, the 3-year survival rates of G-NENs patients with $\mathrm{Ki}-67<20 \%, 20 \%-50 \%$, and $>50 \%$ were $75 \%, 57 \%$, and $54 \%$, respectively (Fig. $2 \mathrm{c}, \mathrm{P}=0.01$ ). In the SSTR2-negative group, the 3-year survival rates of SSTR5-positive and SSTR5-negative were $63 \%$ and $67 \%$, respectively (Fig. 2 d, $P=0.003$ ). The 3-year survival rate of $C D-56$ negative group was significantly lower than that of $C D-56$ positive group ( $36 \%$ vs. $70 \%$, Fig. $2 e, P=0.029$ ). Meanwhile, a subgroup analysis of G3 patients in the tumor grade showed that the 3-year survival rate of G-NENs in the SSTR2-positive group was significantly higher than that in the SSTR2-negative group ( $89 \%$ vs. $57 \%$, Fig. $2 g, P=0.037$ ). Moreover, the 3-year survival rate of the SSTR2-positive group increased by $47 \%$ ( $82 \%$ vs. $35 \%$ ) compared with the SSTR2-negative group, which was statistically significant (Fig. 2f, P=0.002).

In the SSTR5-positive group, the 3-year survival rates of patients with tumor grades of G1, G2, G3 NEN and G3 were $86 \%, 93 \%, 60 \%$ and $56 \%$, respectively (Fig. $2 \mathrm{~h}, \mathrm{P}=0.012$ ). The 3-year survival rates for SSTR2 negative and positive patients were $57 \%$ and $96 \%$, respectively (Fig. $2 \mathrm{i}, \mathrm{P}=0.002$ ). The 3 -year survival rate of CD-56 negative patients was significantly lower than that of CD-56 positive patients $(17 \% \mathrm{vs}$. $89 \%$, Fig. $2 \mathrm{j}, \mathrm{P}=0.000$ ). Moreover, the 3-year survival rates of G-NENs patients with $\mathrm{Ki}-67<20 \%, 20 \%-50 \%$ and $>50 \%$ were $91 \%, 71 \%$ and $64 \%$, respectively(Fig. $2 k, P=0.001$ ), with statistically significant differences.

Meanwhile, a subgroup analysis of G3 patients in the tumor grade showed that the 3-year survival rate of G-NENs in the SSTR5-positive group was slightly lower than that in the negative group (56\% vs. $63 \%$, Fig. $2 \mathrm{~m}, \mathrm{P}=0.001$ ). Moreover, the 3 -year survival rate of the SSTR5 negative group was $7 \%$ lower than that of the SSTR5 positive group( $76 \%$ vs. $83 \%$ ), and the difference was statistically significant (Fig. $2 \mathrm{l}, \mathrm{P}=0.000$ ).

\section{Multivariate Analysis}

Cox proportional risk model was used to analyze all the patients in this study (Table 5). The results showed that SSTR2 and SSTR5 expression were independent prognostic factors for patients with gastric neuroendocrine neoplasms. CD-56 was an independent prognostic factor for gastric neuroendocrine neoplasms patients in the SSTR2 positive expression group, the SSTR2 negative group and the positive SSTR5 group. 
Table 5

Independent Prognostic Factors at Multivariate Analysis by Cox Model in Gastric neuroendocrine neoplasms

\begin{tabular}{|llllll|}
\hline Variable & & $\mathrm{X}^{2}$ value & P-value & HR & $95 \% \mathrm{Cl}$ \\
\hline All cases & Tumor grade & 1.013 & 0.603 & 0.428 & $0.081-2.244$ \\
\cline { 2 - 6 } & SSTR2 & 7.307 & 0.007 & 5.325 & $1.584-17.905$ \\
\hline SSTR5 & 11.097 & 0.001 & 9.899 & $4.34-25.986$ \\
\hline SSTR2 Positive group & KST-67 & 2.755 & 0.252 & 0.368 & $0.113-1.198$ \\
\hline & CD-56 & 16.325 & 0.000 & 11.593 & $3.532-18.058$ \\
\hline KSTR2 negative group & SSTR5 & 0.005 & 0.945 & 0.285 & $0.032-2.588$ \\
\hline & CD-56 & 3.536 & 0.06 & 2.838 & $0.957-8.418$ \\
\hline SSTR5 Positive group & Tumor grade & 0.932 & 0.628 & 0.551 & $0.077-1.23$ \\
\hline & SSTR2 & 2.776 & 0.096 & 4.179 & $0.777-2276$ \\
\hline & CD-56 & 12.215 & 0.000 & 15.232 & $3.307-70.153$ \\
\hline & Ki-67 & 5.748 & 0.056 & 0.195 & $0.051-0.742$ \\
\hline
\end{tabular}

\section{Discussion}

Somatostatin receptor (SSTR) is a glycoprotein with 7 transmembrane segments, which mainly exists in the central system and secretory organs and is mostly related to neuroendocrine neoplasms. Currently, the recognized molecular subtype is SSTR1-5. Since SSTRs is widely used in neuroendocrine tumors, antitumor therapy is commonly used. It was found that SSTR2 was the most commonly express subtype, followed by SSTR5 [8,9]. However, there are few studies on SSTR2 and SSTR5 in gastric neuroendocrine neoplasms (G-NENs). Currently, there are no major case studies on the expression of SSTR2 and SSTR5 in gastric neuroendocrine neoplasms, and the prognostic value of SSTR2 and SSTR5 in patients with gastric neuroendocrine neoplasms has not been clearly reported. Therefore, in this study, the expression levels of SSTR2 and SSTR5 proteins were detected by immunohistochemical S-P method in the tissue samples of 66 patients with gastric neuroendocrine neoplasms and 12 samples of paracancer normal tissues. Then, correlation analysis and survival analysis were conducted based on the clinicopathological data and follow-up data of the patients.

The results of this study showed that the positive expression rate of SSTR2 in gastric neuroendocrine neoplasms tissue specimens and paracancer normal tissue specimens was $48.5 \%$ and $25.0 \%$, respectively, with statistically significant differences. That is, the positive expression rate of SSTR2 
protein in gastric neuroendocrine neoplasms tissues is higher than that in normal tissues, which is consistent with other common neuroendocrine neoplasms tissues reported in the literature. The positive expression rates of SSTR5 in gastric neuroendocrine neoplasms and paracancer normal tissues were $65.2 \%$ and $25.0 \%$, respectively. That is, the positive expression rate of SSTR5 protein in gastric neuroendocrine neoplasms tissues is higher than that in normal tissues.

Cytokeratin (CK) is the main skeletal protein of cells. Studies have shown that CK-8, a member of the cytokeratin (CK) family, is a receptor of plasminogen, which can activate plasminogen and has the effect of reducing the extracellular matrix and basement membrane of tumor. CK-8 is closely related to the metastasis and invasion of tumor. As a member of the superimmune protein family, CD-56 molecule is an isomer of the adhesion molecules of nerve cells and a marker of natural killer lymphocytes (NK). It mediates the interaction between cells and cells, and is mostly associated with acute myeloid leukemia.Studies have also shown that CD-56 has different prognostic significance in AML of different subtypes. $\mathrm{CgA}$ is an acidic protein composed of 439 amino acids, which is mostly located in the dense core granules of neuroendocrine cells and is often used for the detection of neuroendocrine cells [10]. Syn is a membrane protein closely related to the function of cell transmission synapses, which is mostly located in nerve tissues [11]. Ki-67 is a nuclear antigen related to cell proliferation, which is mainly used to evaluate the proliferation activity of various tumor cells. It is currently recognized as the most optimal and convenient biological marker for tumor cell proliferation [12,13]. Ki-67 protein is highly correlated with the development, metastasis and prognosis of malignant tumors, which is an indicator closely related to cell proliferation. In addition, high tumor proliferation is usually associated with tumor invasion and metastasis. So high expression of $\mathrm{Ki}-67$ often indicates poor prognosis of patients with tumor.

In this study, the expression rates of CK-8 and CD-56 were both over $60 \%$, suggesting that gastric neuroendocrine neoplasms have the possibility of highly malignant transformation. The expression rates of $\mathrm{CgA}$ and Syn were $65.9 \%$ and $97.0 \%$, respectively, which also confirmed the high specificity of $\mathrm{CgA}$ and Syn in the detection of gastric neuroendocrine neoplasms. Ki-67 is expressed to different degrees in gastric neuroendocrine neoplasms, which indicates that different G-NENs have different proliferation activities and prognosis. The higher the expression of $\mathrm{Ki}-67$, the worse the prognosis. In order to analyze the clinical significance and prognostic value of SSTR2 and SSTR5 on gastric neuroendocrine neoplasms, this study was divided into SSTR2, SSTR5 positive group and SSTR5 negative group. Immunohistochemical results showed that SSTR2 expression was not significantly correlated with age, gender, tumor site, tumor size, invasion depth, lymph node metastasis, vessel and nerve invasion in GNENs patients, which was consistent with relevant literature reports. SSTR2 expression was closely related to tumor grade, SSTR5 and ki-67 in G-NENs patients, and the difference was statistically significant. Moreover, SSTR2 was negatively associated with the increase of tumor grade and ki-67 while it was positively correlated with the expression of SSTR5. SSTR5 expression was closely related to tumor grade, CD-56, Ki-67 and SSTR2 in G-NENs patients, and the difference was statistically significant. Moreover, SSTR5 is negatively associated with the increase of tumor grade and ki-67 while it was positively correlated with the expression of SSTR2. 
These results indicate that during the whole development process of gastric neuroendocrine neoplasms, the expression levels of SSTR2 and SSTR5 gradually decrease and the inhibitory effect on tumor proliferation is reduced, which is in favor of the growth of gastric neuroendocrine neoplasms. Moreover, the expressions of SSTR2 and SSTR5 complement each other, which are positively correlated. However, in the SSTR5-positive group, the positive rate of CD-56 increased significantly, suggesting that patients with SSTR5-positive expression of gastric neuroendocrine neoplasms have a poor prognosis. It seems to contradict the tumor grade and the expression of $\mathrm{Ki}-67$ in the SSTR5-positive group. But the carcinogenesis of gastric neuroendocrine neoplasms is a complex and dynamic process with the participation of many molecules, which mutual coordinated and restrained each other. Moreover, it is important to note that in this study, tumor grade is divided into four grades (G1, G2, G3 NEN, and G3), which some scholars believe that the $\mathrm{G} 3$ neuroendocrine neoplasms is not a simple neuroendocrine carcinoma, but high proliferation activity of the neuroendocrine tumor and G3 neuroendocrine carcinoma [14]. The research also proved the importance of tumor grade in gastric neuroendocrine neoplasms and rationality.

Vesterinen T et al used immunohistochemistry to detect the expression of SSTR1-5 in lung neuroendocrine tumor specimens and found that the expressions of SSTR2 and SSTR5 highly expressed in lung neuroendocrine tumor, and SSTR2 expression was higher than SSTR5 expression. Survival analysis showed that SSTR2 and SSTR5 were related to their prognosis and SSTR2 was an independent prognostic factor in patients with pulmonary neuroendocrine tumor and improved the prognosis [15]. Lodewijk L et al showed that SSTR2 was related to the prognosis of patients with medullary thyroid cancer [16]. Orlova KV et al revealed that the expression of SSTR2 in merkel cell carcinoma was associated with prognosis [17]. Kiviniemi A showed that SSTR2 is associated with oligodendroglioma in glioma cells and has A good prognosis [18]. Wang Y's study showed that SSTR2 and SSTR5 are the most common SSTR2 and SSTR5 positive expressions in gastrointestinal pancreatic neuroendocrine tumors while SSTR2 and SSTR5 positive expressions can predict the survival rate of patients with GEP-NENs. Meanwhile, the expression of various SSTRS on tumor cells constitutes the basis for the treatment of patients with neuroendocrine tumors with somatostatin.

Therefore, the authors suggest that the expression of SSTR2 and SSTR5 may be a potential indicator of the prognosis of gastric neuroendocrine neoplasms. According to the above literature, SSTR2 and 5 have higher positive expression rates in various neuroendocrine tumors than those in normal tissues, which mostly believe that the expression of SSTR2 is related to the prognosis of patients and improves the prognosis. However, there are few reports on the correlation between SSTR5 and the prognosis of neuroendocrine tumors. In this study, the 3-year survival rate of positive group of SSTR2 and SSTR 5 were $47 \%(82 \%$ vs. $35 \%)$ and $7 \%$ ( $83 \%$ vs. $76 \%$ ), which were higher than those of negative group, respectively, with statistically significant differences. Single factor analysis showed that in the SSTR2 positive group, SSTR5, CD-56 and Ki-67 were closely related to the prognosis of patients with G-NENs. In the SSTR2 negative group, SSTR5 and CD-56 were closely related to the prognosis of patients with G-NENs. In the SSTR5-positive group, tumor grade, SSTR2, CD-56, Ki-67 were closely related to the prognosis of patients 
with G-NENs. All of these suggest that SSTR2 and SSTR5 proteins may have a certain influence on the carcinogenesis and prognosis of gastric neuroendocrine neoplasms.

Cox multivariate analysis showed that among all patients with gastric neuroendocrine neoplasms, SSTR2 and SSTR5 were independent prognostic factors for patients with gastric neuroendocrine neoplasms, and their positive expression suggested improved prognosis, which was consistent with the study results of Vesterinen $T$ et al in lung carcinoid. Meanwhile, CD-56 was an independent factor affecting the prognosis of G-NENs patients in the SSTR2 positive group, SSTR2 negative group and SSTR5 positive group, which suggests that SSTR2 and SSTR5 are closely related to the expression of CD-56. Although CD-56 in patients with G-NENs is rarely studied, the expression of CD-56 in acute myeloid leukemia is more studied, which belongs to the member of the super-immune protein family and has a cellular regulatory effect, indicating poor prognosis of AML $[19,20]$. These also indirectly reflected the positive association between SSTR2, SSTR5 expression and tumor prognosis.

\section{Conclusion}

In summary, tumor development is a complex process involving in multiple factors and many genes. This study shows that SSTR2 and SSTR5 are closely related to the development and prognosis of G-NENs, which suggest improvement of prognosis. Somatostatin and its analogues are widely used in the diagnosis and treatment of neuroendocrine neoplasms. Therefore, the study on the expression and prognostic value of somatostatin subtypes (SSTR2, SSTR5) in G-NENs is helpful for the combination of somatostatin and its analogs with traditional tumor therapy, which provide reliable evidence and promising prospects for tumor diagnosis and treatment.

\section{Declarations}

\section{Acknowledgments}

This study was supported by Department of Gastrointestinal Surgery and Institute of Clinical Medicine, The First Affiliated Hospital, Zhengzhou University and National Natural Science Foundation of China, Grant No. 81201955. Furthermore, this work was supported by the Foundation of Henan Educational Committee (Grant No.19A320080).

\section{Authors' contributions}

Yanwei Ye supervised the study. Yanwei Ye, Chuangfeng Xiao and Yingze Li contributed to the study concept and design. Chuangfeng Xiao. Jingjing Li Yiming Shan and Yingze Li performed experiments and/or acquired data. Chao Han and Wencai Li contributed to the acquisition of samples and clinical data. Yanwei Ye and Chuangfeng Xiao drafted the manuscript. All authors critically reviewed the manuscript for important intellectual content and approved its final version. 


\section{Funding}

The Natural Science Foundation of China (Grant No.81201955) to Yanwei Ye and Foundation of Henan Educational Committee (Grant No.19A320080) to Yanwei Ye.

\section{Availability of data and materials}

All data generated or analyzed during this study are included in this published Article.

\section{Ethics approval and consent to participate}

Informed consent was obtained from all subjects and the study received the approval of the Ethics Committees of the First Affiliated Hospital of Zhengzhou University.

\section{Consent for publication}

Not applicable.

\section{Competing interests}

The authors declare that they have no competing interests.

\section{References}

1. Pawlikowski M, Pisarek H, Winczyk K. Immunohistochemical detection of dopamine D2 receptors in neuroendocrine tumours. Endokrynol Pol. 2011;62(5):388-91.

2. Leijon H, Remes S, Hagström J, Louhimo J, Mäenpää H, Schalin-Jäntti C, et al. Variable somatostatin receptor subtype expression in 151 primary pheochromocytomas and paragangliomas. Hum Pathol. 2019;86(4):66-75.

3. Zalatnai A, Galambos E, Perjési E. Importance of Immunohistochemical Detection of Somatostatin Receptors. Pathol Oncol Res. 2019;25(2):521-5.

4. Kanakis G, Grimelius L, Spathis A, Tringidou R, Rassidakis GZ, Öberg K, et al. Expression of Somatostatin Receptors 1-5 and Dopamine Receptor 2 in Lung Carcinoids: Implications for a Therapeutic Role. Neuroendocrinology. 2015;101(3):211-22.

5. Yang RH, Chu YK. Zollinger-Ellison syndrome: Revelation of the gastrinoma triangle. Radiol Case Rep. 2015;10(1):827.

6. Wang Y, Wang W, Jin K, Fang C, Lin Y, Xue L, et al. Somatostatin receptor expression indicates improved prognosis in gastroenteropancreatic neuroendocrine neoplasm, and octreotide long-acting 
release is effective and safe in Chinese patients with advanced gastroenteropancreatic neuroendocrine tumors. Oncol Lett. 2017;13(3):1165-74.

7. Niederle MB, Hackl M, Kaserer K, Niederle B. Gastroenteropancreatic neuroendocrine tumours: the current incidence and staging based on the WHO and European Neuroendocrine Tumour Society classification: an analysis based on prospectively collected parameters. Endocr Relat Cancer. 2010;17(4):909-18.

8. Mai R, Kaemmerer D, Träger T, Neubauer E, Sänger J, Baum RP, et al. Different somatostatin and CXCR4 chemokine receptor expression in gastroenteropancreatic neuroendocrine neoplasms depending on their origin. Sci Rep. 2019;9(1):4339.

9. Yenıay L, Gürcü B, Ünalp Ö,Yilmaz F, Nart D, Sözbılen M, et al. Prognostic value of somatostatin receptor-2 positivitiy in gastroenteropancreatic neuroendocrine tumors in reference to known prognostic factors. Turk J Gastroenterol. 2012;23(6):736-40.

10. Zhang C, Huang Y, Long J, Yao X, Wang J, Zang S, et al. Serum chromogranin A for the diagnosis of gastroenteropancreatic neuroendocrine neoplasms and its association with tumour expression. Oncol Lett. 2019;17(2):1497-504.

11. Wang YH, Yang QC, Lin Y, Xue L, Chen MH, Chen J. Chromogranin A as a marker for diagnosis, treatment, and survival in patients with gastroenteropancreatic neuroendocrine neoplasm. Medicine (Baltimore). 2014;93(27):e247.

12. Foltyn W, Zajęcki W, Marek B, Kajdaniuk D, Siemińska L, Zemczak A, et al. The value of the Ki-67 proliferation marker as a prognostic factor in gastroenteropancreatic neuroendocrine tumours. Endokrynol Pol. 2012;63(5):362-6.

13. Strosberg J, Kvols L. Antiproliferative effect of somatostatin analogs in gastroenteropancreatic neuroendocrine tumors. World J Gastroenterol. 2010;16(24):2963-70.

14. Siddiqa A, Adel H, Khan SA, Huda F, Sattar A. Gastrointestinal and pancreatic neuroendocrine tumours and carcinomas; a review of rare tumour type. J Pak Med Assoc. 2019;69(4):533-40.

15. Vesterinen T, Leijon H, Mustonen H, Remes S, Knuuttila A, Salmenkivi K, et al. Somatostatin Receptor Expression Is Associated With Metastasis and Patient Outcome in Pulmonary Carcinoid Tumors. J Clin Endocrinol Metab. 2019;104(6):2083-2093.

16. de Vries LH, Lodewijk L, Willems SM, etDreijerink KMA, de Keizer B, van Diest PJ, et al. SSTR2A expression in medullary thyroid carcinoma is correlated with longer survival. Endocrine. 2018;62(3):639-47.

17. Orlova KV, Delektorskaya VV, Vishnevskaya YV, et Kondratieva TT, Orel NF, Markovich AA, et al. Somatostatin receptor type 2 expression in Merkel cell carcinoma as a prognostic factor. J Eur Acad Dermatol Venereol. 2018;32(6):e236-e237.

18. Kiviniemi A, Gardberg M, Kivinen K, Posti JP, Vuorinen V, Sipilä J, et al. Somatostatin receptor 2A in gliomas: Association with oligodendrogliomas and favourable outcome. Oncotarget. 2017;8(30):49123-32. 
19. Hess LU, Martrus G, Ziegler AE, Langeneckert AE, Salzberger W, Goebels $H$, et al.The Transcription Factor Promyelocytic Leukemia Zinc Finger Protein Is Associated With Expression of Liver-Homing Receptors on Human Blood CD56 bright Natural Killer Cells. Hepatol Commun. 2020;4(3):409-24.

20. Aref S, Azmy E, El-Bakry K, Ibrahim L, Mabed M. Prognostic impact of CD200 and CD56 expression in adult acute lymphoblastic leukemia patients. Hematology. 2018;23(5):263-70.

\section{Figures}

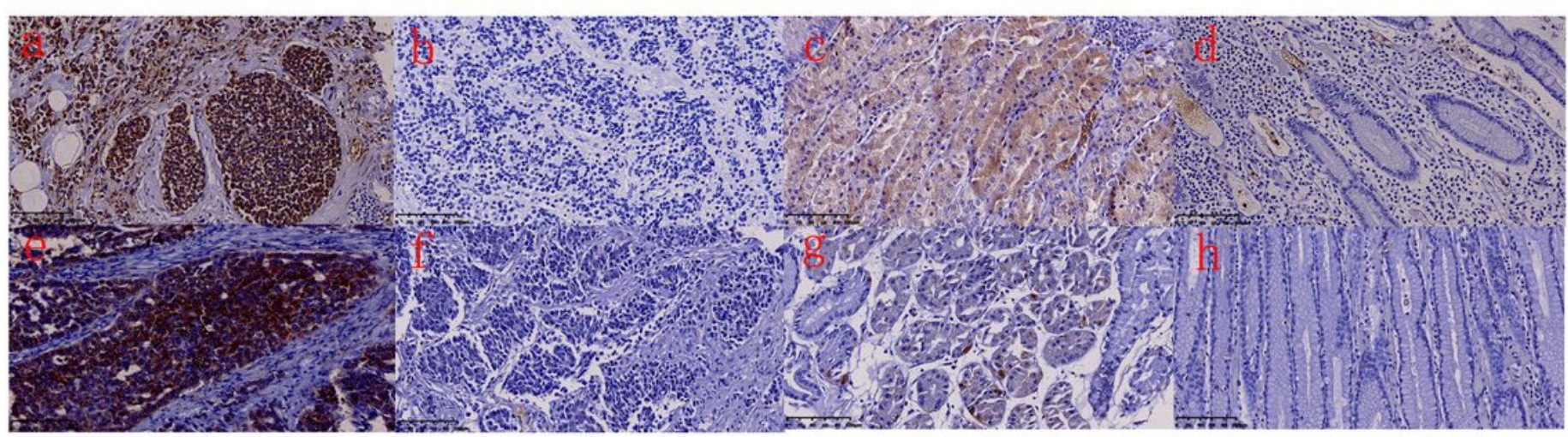

\section{Figure 1}

a-d shows immunohistochemical sections of SSTR2 expression in gastric neuroendocrine neoplasm and paracancer tissues. All immunohistochemical pictures were amplifified 200-fold. a positive expression of SSTR2 in gastric neuroendocrine tissue. b negative expression of SSTR2 in gastric neuroendocrine tissue. c positive expression of SSTR2 in paracancer normal tissue. $d$ negative expression of SSTR2 in paracancer normal tissue. Fig. 1 e-h shows immunohistochemical sections of SSTR5 expression in gastric neuroendocrine neoplasm and paracancer tissues. e positive expression of SSTR5 in gastric neuroendocrine tissues. $f$ negative expression of SSTR5 in gastric neuroendocrine tissues. g positive expression of SSTR5 in para-cancer normal tissues. h negative expression of SSTR5 in paracancer normal tissues. 

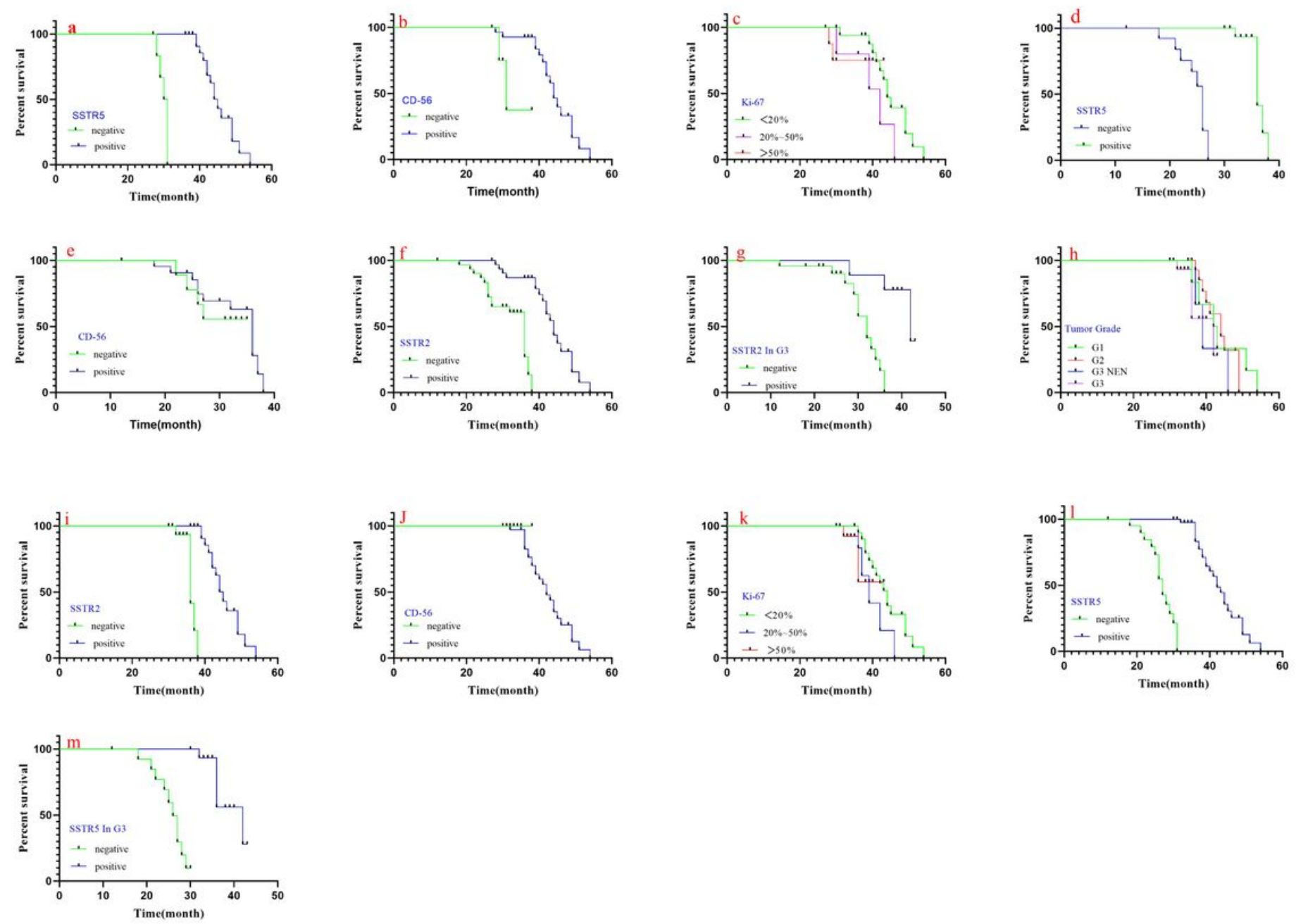

\section{Figure 2}

The survival curves of the patients with gastric neuroendocrine neoplasm by Kaplan-Meier method (log rank test). In the SSTR2-positive group, there were signifificant differences in SSTR5 expression (2a, $P=0.000$ ), $C D-56$ expression ( $2 b, P=0.000$ ), $K i-67$ expression ( $2 c, P=0.01$ ). In the SSTR2-negative group, significant differences existed in SSTR5 expression ( $2 d, P=0.003), C D-56$ expression $(2 e, P=0.029)$. The survival difference was showed between the SSTR2-positive group and the SSTR2-negative group (2f, $P=0.002)$. The survival difference was revealed between SSTR2-positive and SSTR2-negative gastric neuroendocrine tumor in the tumor grade $\mathrm{G} 3$ subgroup ( $2 \mathrm{~g}, \mathrm{P}=0.037)$. In the SSTR5-positive group, there were signifificant differences in tumor grades $(2 \mathrm{~h}, \mathrm{P}=0.012)$, SSTR2 expression ( $2 \mathrm{i}, \mathrm{P}=0.002), \mathrm{CD}-56$ expression (2j, $\mathrm{P}=0.000)$, $\mathrm{Ki}-67$ expression ( $2 \mathrm{k}, \mathrm{P}=0.001)$. The survival difference was showed between SSTR5-positive and SSTR5-negative in the tumor grade G3 subgroup $(2 \mathrm{~m}, \mathrm{P}=0.001)$. The survival difference was showed between the SSTR5-positive and SSTR5-negative group ( 2 l, $P=0.000)$. 